\title{
Srlp is crucial for the self-renewal and differentiation of germline stem cells via RpL6 signals in Drosophila testes
}

\author{
Jun Yu ${ }^{1,2}$, Yidan Yan ${ }^{1,2}$, Xiaojin Luan ${ }^{1,2}$, Chen Qiao ${ }^{3}$, Yuanyuan Liư ${ }^{4}$ Dan Zhao ${ }^{2,5}$, Bing Xie ${ }^{1}$, Qianwen Zheng ${ }^{1,2}$,

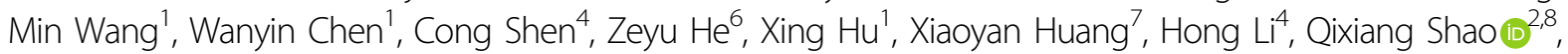 \\ Xia Chen ${ }^{1,2}$, Bo Zheng ${ }^{4}$ and Jie Fang ${ }^{1}$
}

\begin{abstract}
Self-renewal and differentiation in germline stem cells (GSCs) are tightly regulated by the stem cell niche and via multiple approaches. In our previous study, we screened the novel GSC regulatory gene Srlp in Drosophila testes. However, the underlying mechanistic links between Srlp and the stem cell niche remain largely undetermined. Here, using genetic manipulation of the Drosophila model, we systematically analyze the function and mechanism of Srlp in vivo and in vitro. In Drosophila, Srlp is an essential gene that regulates the self-renewal and differentiation of GSCs in the testis. In the in vitro assay, Srlp is found to control the proliferation ability and cell death in S2 cells, which is consistent with the phenotype observed in Drosophila testis. Furthermore, results of the liquid chromatographytandem mass spectrometry (LC-MS/MS) reveal that RpL6 binds to Srlp. Srlp also regulates the expression of spliceosome and ribosome subunits and controls spliceosome and ribosome function via RpL6 signals. Collectively, our findings uncover the genetic causes and molecular mechanisms underlying the stem cell niche. This study provides new insights for elucidating the pathogenic mechanism of male sterility and the formation of testicular germ cell tumor.
\end{abstract}

\section{Introduction}

Stem cells are undifferentiated populations with the remarkable potential of self-renewal and differentiation. The stem cell niche, a key microenvironment that regulates stem cell behaviors, supports two distinct adult stem cell populations: germline stem cells (GSCs) and cyst stem cells $(\mathrm{CySCs})^{1-3}$. In Drosophila testes, GSCs asymmetrically divide to generate one cell that retains stemness

\footnotetext{
Correspondence: Xia Chen (chenxia_0511@126.com) or Bo Zheng (mansnoopy@163.com) or Jie Fang (fangjie070@163.com)

${ }^{1}$ Department of Gynecology, The Affiliated Hospital of Jiangsu University, Jiangsu University, Zhenjiang Jiangsu 212001, China

${ }^{2}$ Reproductive Sciences Institute of Jiangsu University, Zhenjiang Jiangsu

212001, China

Full list of author information is available at the end of the article.

These authors contributed equally: Jun Yu, Yidan Yan, Xiaojin Luan, Chen Qiao, Yuanyuan Liu.
}

Edited by E. Baehrecke and a gonialblast that proliferates and differentiates ${ }^{2}$. The gonialblast undergoes four rounds of transit-amplifying (TA) spermatogonial divisions to generate a 16-cell spermatogonia cluster in which individual germ cells are connected by ring canals and a branched fusome ${ }^{4}$. Somatic cells, including apical hubs and CySCs, form the stem cell environment for neighboring GSCs, and CySCs have been proposed to be a source of instructive selfrenewal signals ${ }^{5}$. CySCs provide the environment necessary to trigger GSC differentiation by the non-cellautonomous approach ${ }^{6}$.

Early germ cells have been shown to be tightly controlled by niche signaling. Hub cells secrete unpaired (Upd) and hedgehog (Hh) proteins. Upd binds with Domeless (Dome) and activates the Janus kinase/signal transducer and the activator transcription (JAK/STAT) pathway in both GSCs and CySCs, and maintains their

\section{(c) The Author(s) 2019}

(c) (i) Open Access This article is licensed under a Creative Commons Attribution 4.0 International License, which permits use, sharing, adaptation, distribution and reproduction c. in any medium or format, as long as you give appropriate credit to the original author(s) and the source, provide a link to the Creative Commons license, and indicate if changes were made. The images or other third party material in this article are included in the article's Creative Commons license, unless indicated otherwise in a credit line to the material. If material is not included in the article's Creative Commons license and your intended use is not permitted by statutory regulation or exceeds the permitted use, you will need to obtain permission directly from the copyright holder. To view a copy of this license, visit http://creativecommons.org/licenses/by/4.0/. 
self-renewal ability ${ }^{7,8}$. Hh activates the $\mathrm{Hh}$ signaling pathway in CySCs, and is required for the maintenance of $\mathrm{CySCs}^{9}$. Two BMP-like molecules expressed in somatic cells, decapentaplegic (Dpp) and glass bottom boat (Gbb), are required for GSC maintenance and repress the differentiation factor bag-of-marbles (Bam) by bone morphogenetic protein (BMP) signaling ${ }^{10}$. Together with its regulator, benign gonial cell neoplasm (Bgcn), Bam is required for spermatogonia to transition from proliferation to differentiation ${ }^{10-12}$. Mutations in bam or $b g c n$ result in germ cell tumors with extensive accumulation of undifferentiated germ cells ${ }^{13,14}$. Bam interacts with Bgcn and tumorous testis (Tut) to repress Mei-P26 expression, establishing a regulatory feedback loop that governs the proliferation of spermatogonia ${ }^{15,16}$.

Drosophila provides a simple system to investigate the complex genetic basis and related molecular mechanisms of biological events in reproduction ${ }^{17-19}$. Previously, a large-scale in vivo RNA interference (RNAi) screening in fly ovaries revealed the presence of a regulatory network involved in the self-renewal and differentiation of $\mathrm{GSCs}^{20}$. In the testis screen, $\mathrm{Yu}$ et al. ${ }^{17}$ found that protein synthesis and degradation, especially spliceosome and ribosome, were essential in the regulation of GSC homeostasis in fly testes.

CG5844 has been identified as a candidate GSC factor with its regulatory mechanism unclear. In this study, we named CG5844 gene as Spliceosome-Ribosome Linker Protein (Srlp). Using in vivo and in vitro approaches, we systematically analyze the function and mechanism of Srlp in Drosophila. Here, we found that Srlp gene is essential for the self-renewal and differentiation of GSCs in Drosophila testis and increases proliferation and apoptosis in S2 cells. Moreover, Srlp regulates spliceosome and ribosome function via ribosomal protein L6 (RpL6) signals. In conclusion, the findings of this study will provide new insights into the mechanism underlying the stem cell niche.

\section{Results}

\section{Srlp deficiency causes GSC self-renewal and differentiation defects}

To determine the function of Srlp in Drosophila testes, we generated Srlp knockout flies using nos-cas9/CRISPR, resulting in a 335-bp deletion (264 bp in the coding sequence (CDS) region) and a code shift (Fig. S1a). The Srlp deletion in Drosophila was confirmed by PCR and sequencing (Fig. S1b and S1c). The homozygous Srlp mutation was lethal $\left(\mathrm{Srlp}^{--}\right)$, while the heterozygous $\mathrm{Srlp}$ mutation $\left(\mathrm{Srlp}^{-/+}\right)$was viable and flies with the heterozygous mutation were fertile (Fig. S1d).

To determine the in vivo function of Srlp in Drosophila testes, we generated a UAS/Gal4-mediated RNAi assay to test the loss of Srlp function using two different Gal4s
(nos-Gal4 and tj-Gal4) that were mainly expressed in the stem cell niche ${ }^{17}$. Results of the immunofluorescence staining and confocal microscopic imaging of marker proteins revealed specific defects at the testicular apex. Srlp knockdown in early germ cells using nos-Gal4 caused tiny testes and complete absence of germ cells (cells were Vasa and TUNEL (terminal deoxynucleotidyl transferasemediated dUTP-biotin nick-end labeling) negative), followed by accumulation of cyst cells (Fig. 1a and Fig. S2a). Moreover, Srlp knockdown driven by tj-Gal4 led to dysfunction of normal cyst cells and accumulation of undifferentiated germ cells, which ultimately developed into testis tumors (Fig. 1b, c), indicating that the Srlp gene is required for germ cell formation and cyst cell organization. Furthermore, these undifferentiated germ cells had proliferation and apoptosis ability (TUNEL and PH3 positive; Fig. S2b and S2c) without normal niche environments (Zfh1 positivity with abnormal pattern, no Eya-positive cyst cells, and no FasIII-positive hub cells; Fig. 1b, c).

\section{Srlp regulates proliferation and apoptosis in Drosophila S2 cells}

To further explore the function of Srlp in vitro, Srlp expression was silenced using two small interfering RNA (siRNAs; siSrlp-303 and siSrlp-684). Results of the quantitative reverse transcription-polymerase chain reaction (qRT-PCR) analysis revealed that siRNA suppression reduced the expression of Srlp messenger RNA (mRNA) in Drosophila S2 cells (Fig. 2a).

As down-regulation of Srlp expression caused defects in the self-renewal and differentiation of GSCs in Drosophila, we investigated whether Srlp was involved in proliferation and apoptosis. Importantly, both PH3-positive and TUNEL-positive cells significantly increased in siSrlp$684(150 \mathrm{nM})$ S2 cells (Fig. 2b-e). Similar results were obtained by flow cytometry, reflecting an increased apoptosis ratio in cells following Srlp knockdown (Fig. 2f). Cell counting kit-8 (CCK-8 kit) was then used to detect whether the growth of S2 cells was affected by treatment with Srlp siRNA. The results indicated that knockdown of Srlp in S2 cells dramatically reduces cell growth (Fig. 2g).

Next, we examined whether Srlp overexpression could lead to malfunctions in proliferation and apoptosis in S2 cells. We generated a UAS-Srlp-3xHA CDS clone driven by ub-Gal4. For the transient expression of Srlp, S2 cells were transfected with $0.2,0.4$, and $0.8 \mu \mathrm{g}$ of plasmid separately. According to the results, the phenotype can only be observed when transfecting with $0.8 \mu \mathrm{g}$ of plasmid (Fig. S3). Therefore, we chose $0.8 \mu$ g of plasmid for the transient expression of Srlp. Up-regulation of Srlp expression was confirmed at the transcription and translation levels (Fig. S4). Both PH3- and TUNEL-positive cells increased dramatically in S2 cells overexpressing Srlp 
a

\begin{tabular}{|c|c|c|c|}
\hline \multicolumn{4}{|c|}{ Nos-Gal4 } \\
\hline Vasa & Eya & De-cad & Verge \\
\hline
\end{tabular}
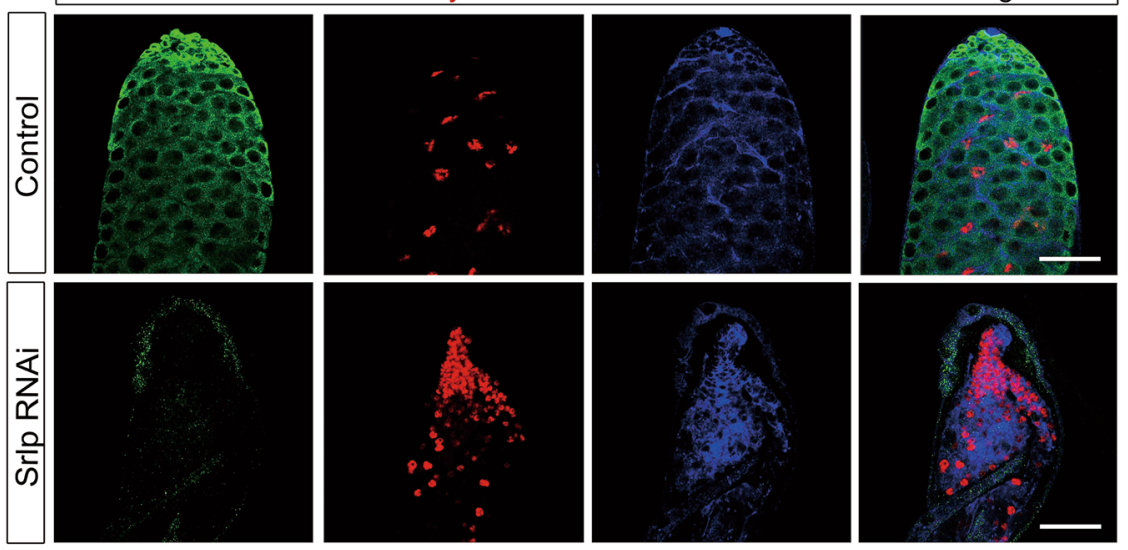

b
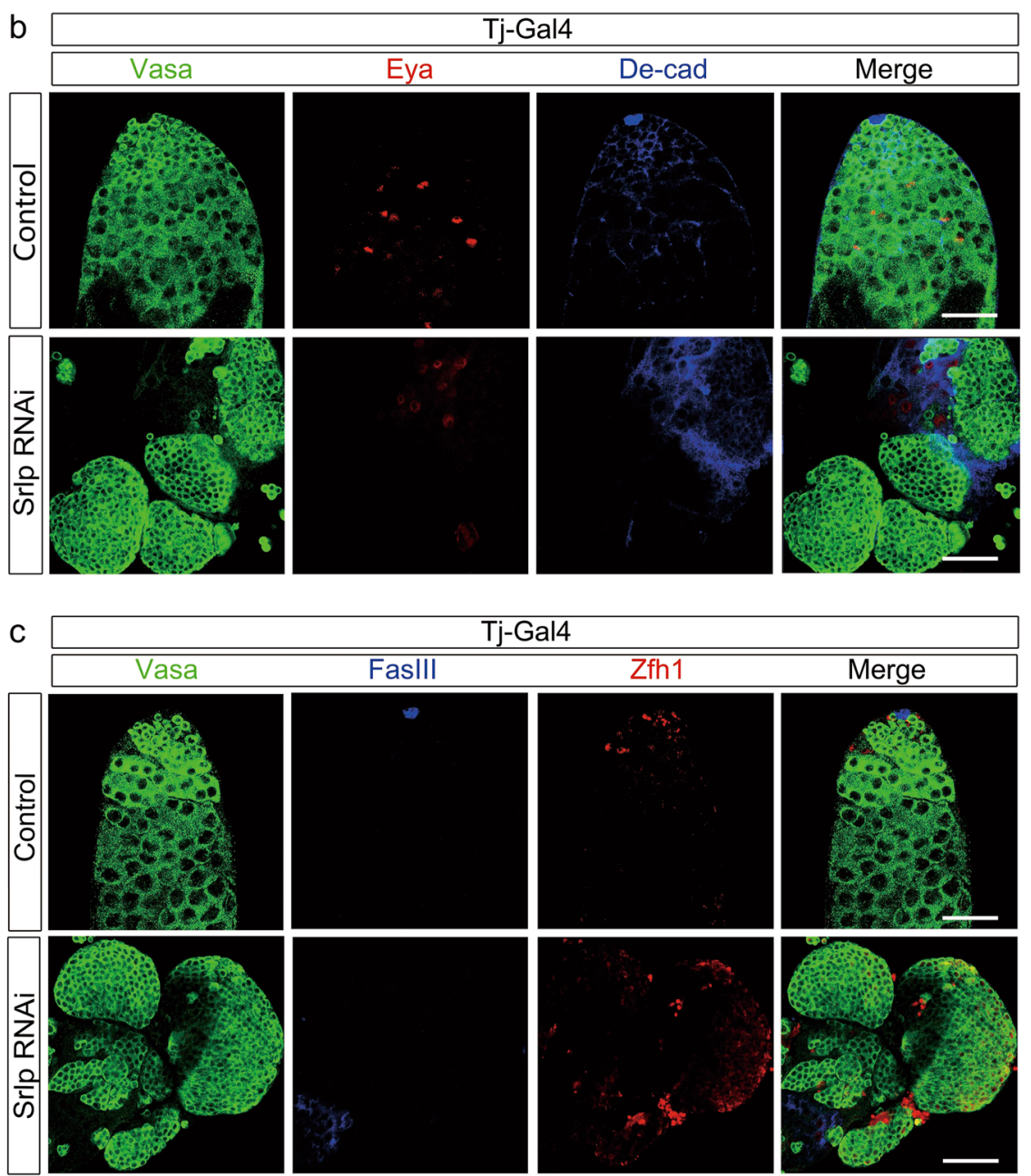

Fig. 1 Srlp is required for the self-renewal and differentiation of germline stem cells (GSCs) in Drosophila testes. a Distribution of germ cells and somatic cells in the apex of the testis of control and nos>Srlp RNA interference (RNAi) flies. $\mathbf{b}$ Distribution of germ cells and somatic cells in the apex of the testis of control and tj>Srlp RNAi flies. c Distribution of germ cells and somatic cells in the apex of the testis of control and tj>Srlp RNAi flies. Immunostaining using anti-Vasa (green), anti-Eya (red in $\mathbf{a}, \mathbf{b}$ ), anti-DE-cad (blue in $\mathbf{a}, \mathbf{b}$ ), anti-Zfh1 (red in c), and anti-Faslll (blue in c) antibodies. Scale bar: $50 \mu \mathrm{m}$ 


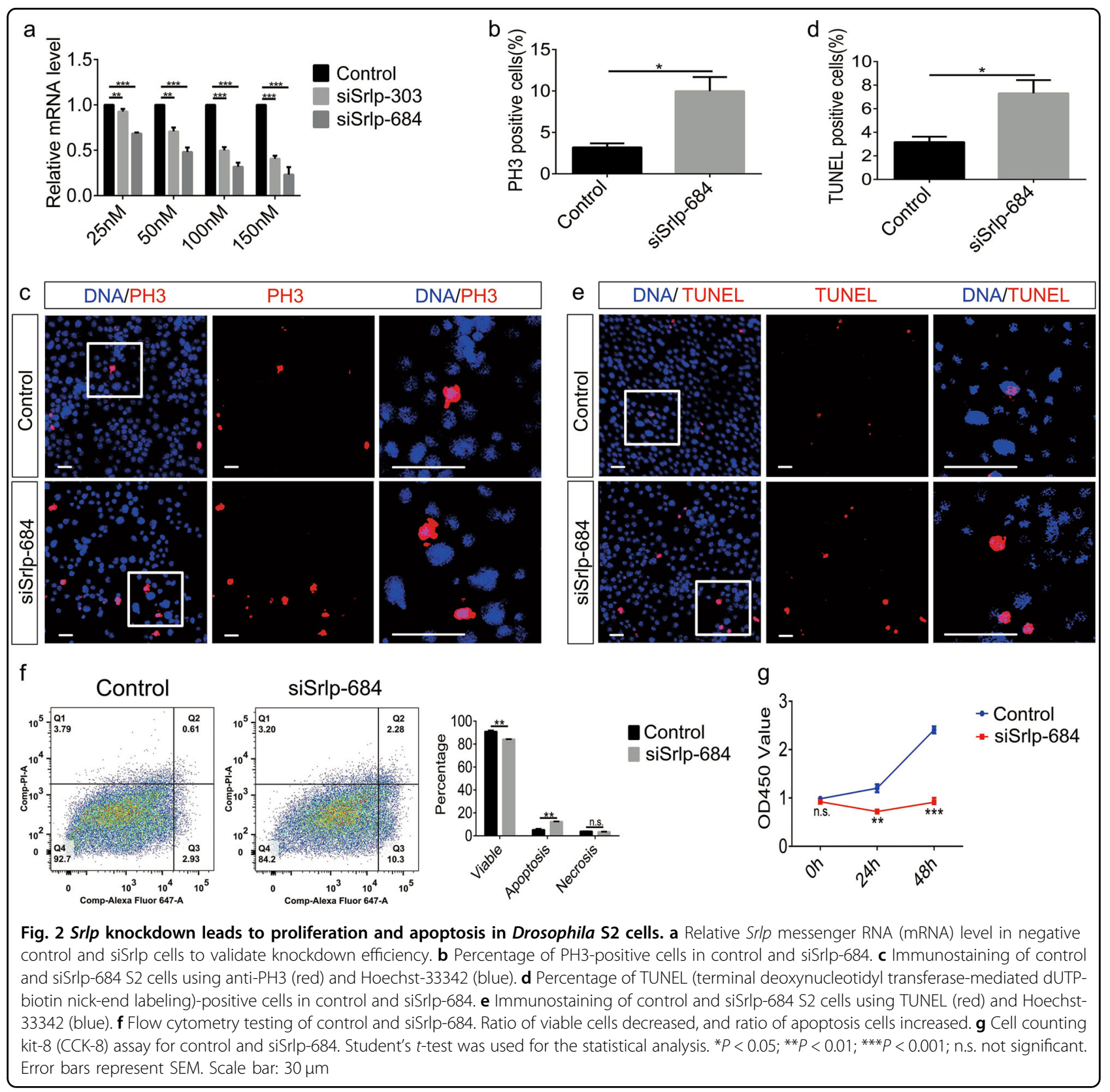

(Fig. 3a-d), and their signals were even stronger than corresponding signals in siSrlp-684 $(150 \mathrm{nM})$. Results of the flow cytometry showed that Srlp overexpression greatly reduced the ratio of viable cells and increased the apoptosis ratio (Fig. 3e). Results of the CCK-8 assay revealed that Srlp overexpression dramatically reduced the cell growth of $\mathrm{S} 2$ cells (Fig. 3f). Taken together, these results indicated that Srlp regulates cell proliferation and apoptosis.

\section{Srlp can rescue proliferation but not cell death}

We conducted two independent Srlp rescue assays in S2 cells to further confirm their phenotype. Results of the
qRT-PCR analysis indicated that Srlp overexpression recovered the Srlp mRNA expression of siSrlp-684 (Fig. 4a). Immunofluorescence staining showed that cell proliferation ability (indicated by $\mathrm{PH} 3$ positivity) could be rescued by Srlp overexpression (Fig. 4b, c). However, the TUNEL-positive cell ratio of Srlp siRNA could not be rescued by Srlp overexpression; on the contrary, it was enhanced by overexpression, reflecting a superposition effect for cell survival defects (Fig. 4d, e). Component analysis of cell death by flow cytometry showed that Srlp overexpression in cells silenced by siSrlp-684 reduced the number of viable cells and dramatically increased the numbers of apoptotic and necrotic cells (Fig. 4f). 

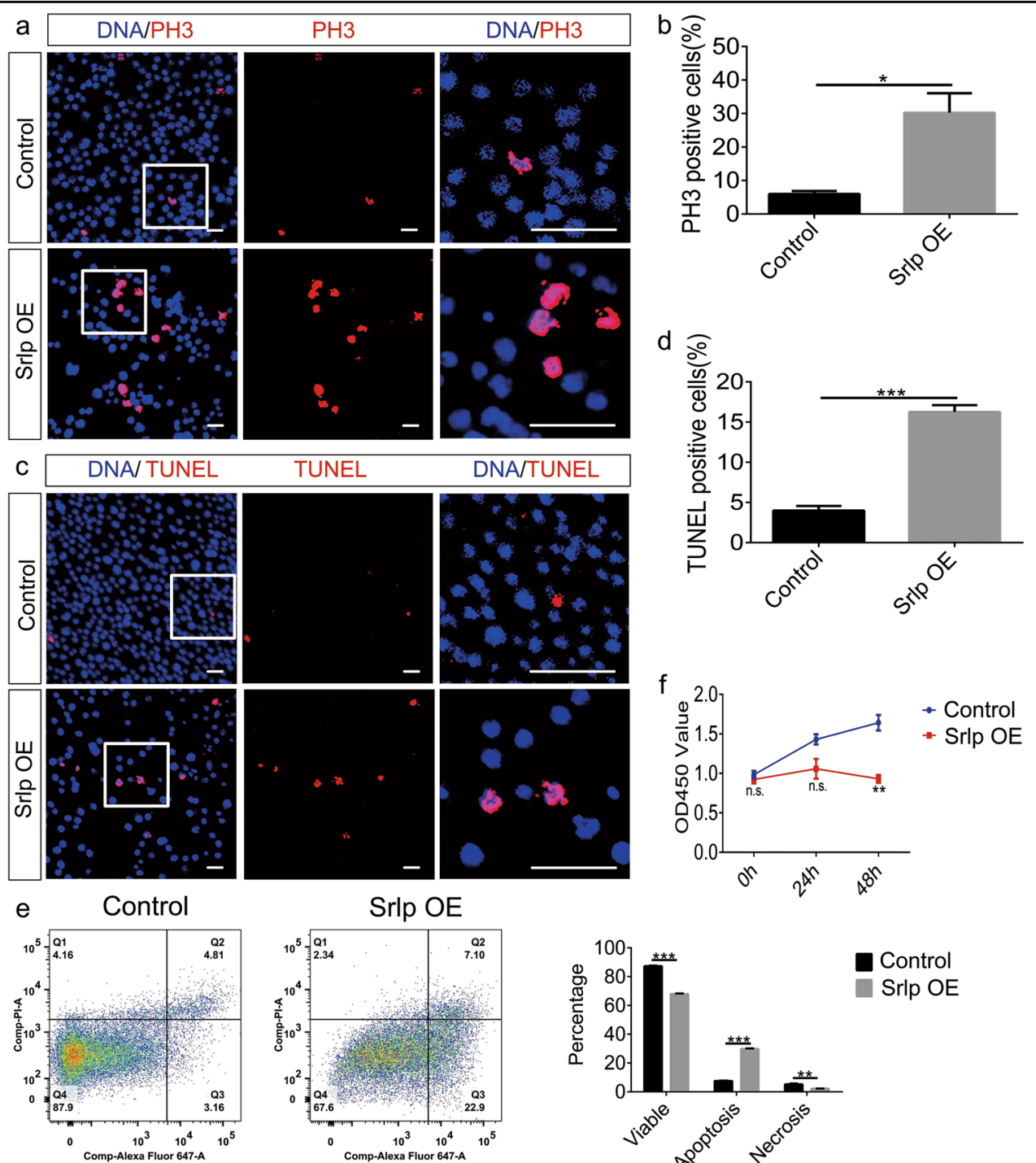

\section{Srlp OE}
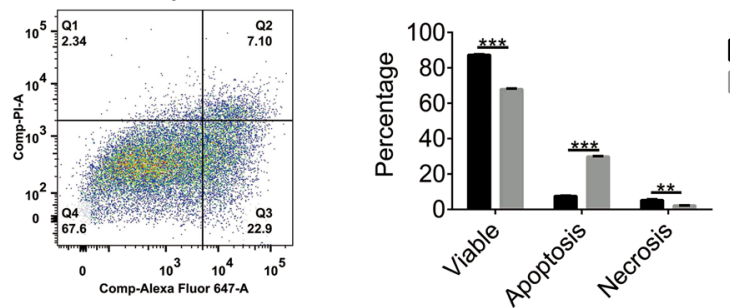

Control

Srlp OE

Fig. 3 Srlp overexpression increases proliferation ability and cell death in Drosophila S2 cells. a Immunostaining of control and Srlpoverexpressing S2 cells using anti-PH3 (red) and Hoechst-33342 (blue). b Percentage of PH3-positive cells in the control and Srlp-overexpression group. c Immunostaining of the control and Srlp-overexpressing S2 cells by TUNEL (terminal deoxynucleotidyl transferase-mediated dUTP-biotin nick-end labeling) (red) and Hoechst-33342 (blue). $\mathbf{d}$ Percentage of TUNEL-positive cells in the control and Srlp-overexpression groups. e Flow cytometry of control and Srlp-overexpressing cells. The ratio of viable cells dramatically decreased while that of apoptosis cells significantly increased. f Cell counting kit-8 (CCK-8) assay of the control and Srlp-overexpressing S2 cells. Student's $t$-test was used for the statistical analysis. ${ }^{*} P<0.05 ;{ }^{* *} P<$ 0.01 ; ${ }^{* *} P<0.001$; n.s. not significant. Error bars represent SEM. Scale bar: $30 \mu \mathrm{m}$

Interestingly, Srlp knockdown could partially rescue the Srlp expression of overexpressed Srlp, and similar results were observed in the Srlp overexpression/siSrlp-684 rescue assay (Fig. S5). Taken together, our data indicated that Srlp rescue assays restored the proliferation ability but accelerated cell death.

\section{Ribosomes were enriched in the regulatory network of Srlp-binding proteins}

To explore the regulatory network of Srlp-binding proteins, we pulled down Srlp-HA fusion proteins with HA beads and identified Srlp-related binding proteins by liquid chromatography-tandem mass spectrometry (LCMS/MS). Thereafter, we identified 205 Srlp-binding proteins in Drosophila melanogaster (Fig. 5a and Table S1). The ten Srlp-binding proteins with the highest scores are listed in Fig. 5a. Bioinformatics analysis was performed by the OmicsBean data integration analysis platform, and classified according to the biological process, cellular component, and molecular function categories. Figure S6 shows the top 20 enriched and significantly different terms based on gene ontology (GO) analysis. In the biological process category, translation, biosynthetic and metabolic processes, and mitotic spindle elongation were 

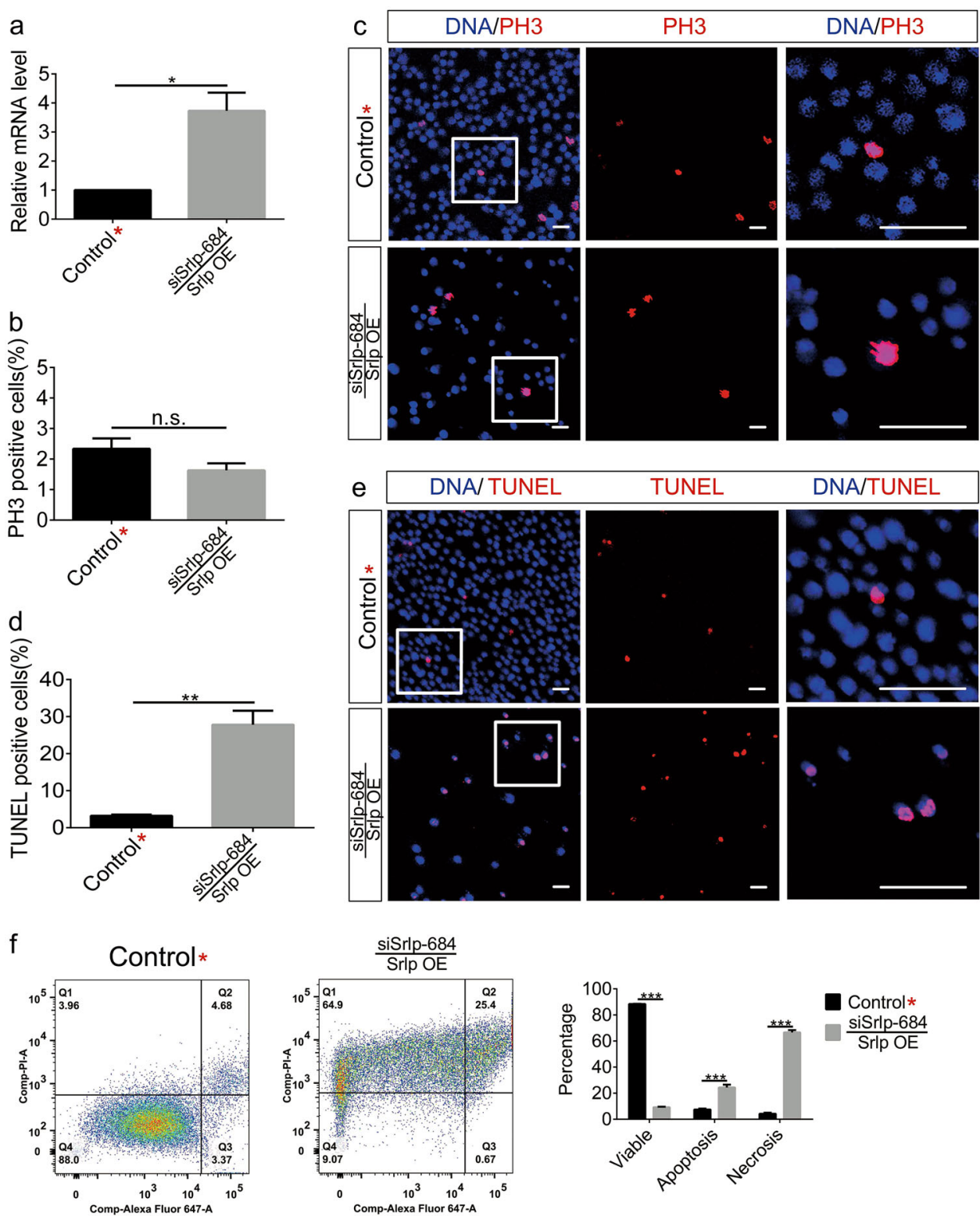

Fig. 4 Rescue experiments of the Srlp knockdown phenotype. a Relative Srlp messenger RNA (mRNA) level of control* and siSrlp-684/Srlpoverexpressing S2 cells. b Percentage of PH3-positive cells in the control* and siSrlp-684/Srlp overexpression groups. c Immunostaining of the control* and siSrlp-684/Srlp-overexpressing S2 cells by anti-PH3 (red) and Hoechst-33342 (blue). d Percentage of TUNEL (terminal deoxynucleotidyl transferase-mediated dUTP-biotin nick-end labeling)-positive cells in control* and siSrlp-684/Srlp overexpression groups. e Immunostaining of control* and siSrlp-684/Srlp-overexpressing S2 cells using TUNEL (red) and Hoechst-33342 (blue). f Flow cytometry test of the control* and siSrlp-684/ Srlp overexpression groups. The ratio of viable cells dramatically decreased while the ratios of apoptotic and necrotic cells significantly increased. Student's $t$-test was used for the statistical analysis. ${ }^{*} P<0.05$; ${ }^{*} P<0.01$; ${ }^{* *} P<0.001$; n.s. not significant. Error bars represent SEM. Scale bar: $30 \mu \mathrm{m}$

mainly enriched in Srlp-binding proteins. In the context of cellular component, cytoplasm, ribosome, and ribonucleoprotein complex were involved in Srlp-binding proteins. Moreover, in the molecular function category, Srlp was found to be related with the structural constituent of ribosome, structural molecular activity, isomerase activity, and RNA binding. Enrichment analysis using the KEGG (Kyoto Encyclopedia of Genes and Genomes) pathway and protein-protein interaction (PPI) demonstrated that the identified Srlp-binding proteins were involved in the ribosome signaling pathway with high confidence $(p<0.01)$, indicating the significant roles of crosstalk between Srlp and the ribosome complex (Fig. 5b, c). 
a
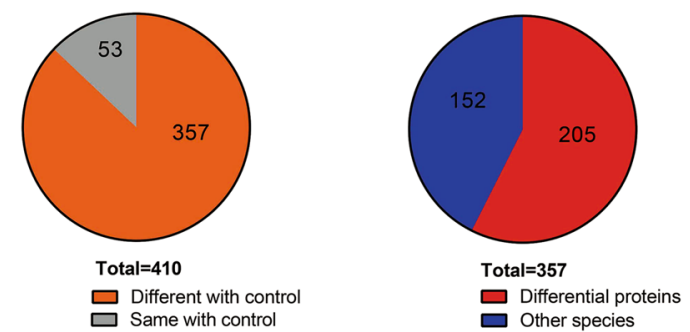

\begin{tabular}{lllllll}
\hline GI NO & Score & Mass & Matches & Sequences & emPAl & Protein description \\
\hline gi|7299632|gb|AAF54816.1| & 878 & 41935 & $89(37)$ & $22(14)$ & 5.17 & Srip \\
gi|7302470|gb|AAF57555.1| & 373 & 50571 & $42(22)$ & $11(8)$ & 0.66 & beta-Tubulin at 56D, isoform B \\
gi|23172610|gb|AAN14192.1| & 305 & 23859 & $16(10)$ & $7(4)$ & 1.51 & ribosomal protein S8, isoform C \\
gi|17137654|ref|NP_477421.1| & 265 & 46861 & $27(11)$ & $13(7)$ & 0.61 & enolase, isoform A \\
gi|7298512|gb|AAF53731.1| & 226 & 25071 & $5(4)$ & $3(2)$ & 0.28 & CG10641 \\
gi|7297772|gb|AAF53022.1| & 225 & 30531 & $22(11)$ & $12(6)$ & 1.06 & porin, isoform A \\
gi|7296991|gb|AAF52262.1| & 225 & 71371 & $21(8)$ & $15(7)$ & 0.37 & lamin, isoform A \\
gi|7298302|gb|AAF53532.1| & 212 & 47179 & $12(7)$ & $7(4)$ & 0.31 & calcium-binding protein 1 \\
gi|7303809|gb|AAF58856.1| & 190 & 21630 & $9(7)$ & $5(3)$ & 0.78 & seele \\
gi|21645522|gb|AAM71060.1| & 190 & 28324 & $11(5)$ & $7(4)$ & 0.74 & 14-3-3zeta, isoform D \\
\hline
\end{tabular}

b

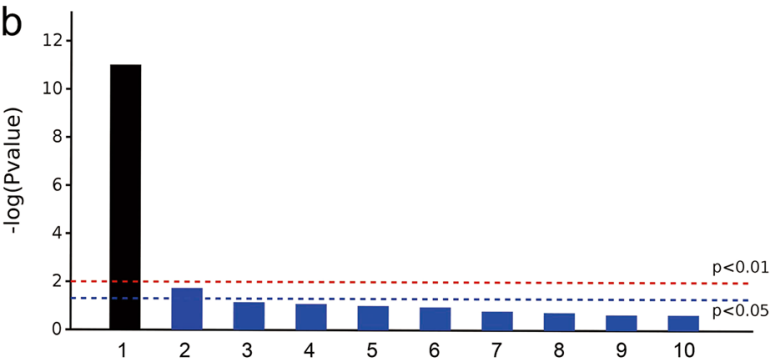

\begin{tabular}{cl}
\hline No. & Pathway Name \\
\hline 1 & Ribosome \\
2 & Protein processing in endoplasmic reticulum \\
3 & Carbon metabolism \\
4 & Proteasome \\
5 & Glycolysis / Gluconeogenesis \\
6 & Phagosome \\
7 & Biosynthesis of amino acids \\
8 & Non-homologous end-joining \\
9 & Endocytosis \\
10 & Phototransduction - fly \\
\hline
\end{tabular}

C

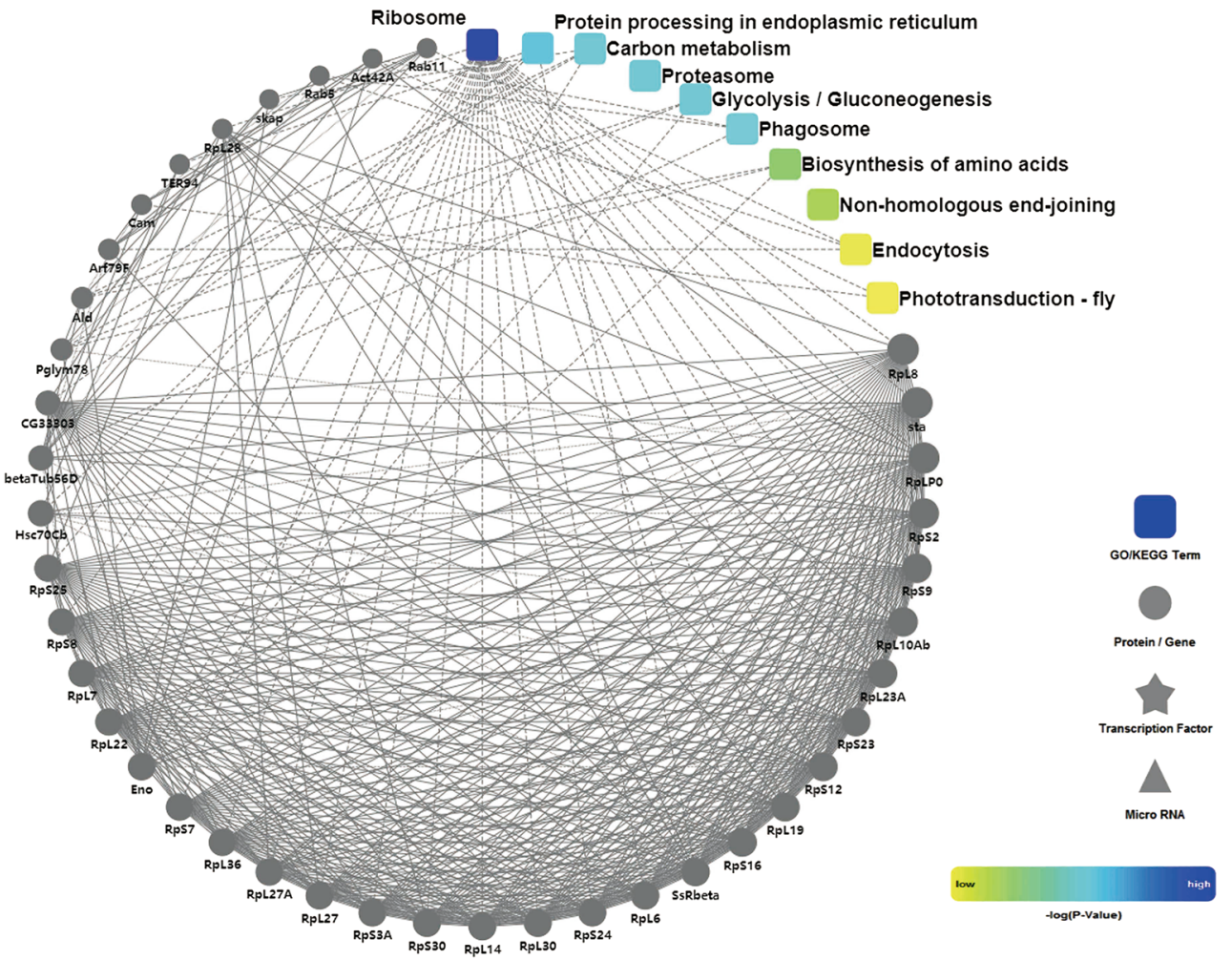

Fig. 5 Characteristics of Srlp-binding proteins in Drosophila melanogaster. a Identification of Srlp-binding proteins. b KEGG (Kyoto Encyclopedia of Genes and Genomes) pathway analysis of identified Srlp-binding proteins in Drosophila S2 cells. c Protein-protein interaction (PPI) analysis of identified Srlpbinding proteins from KEGG pathway analysis in Drosophila S2 cells. Dots represent genes/proteins, and rounded rectangles represent biological processes, cell localization, molecular functions or signaling pathways. Solid lines represent verified interactions, and dashed lines represent predicted interactions 

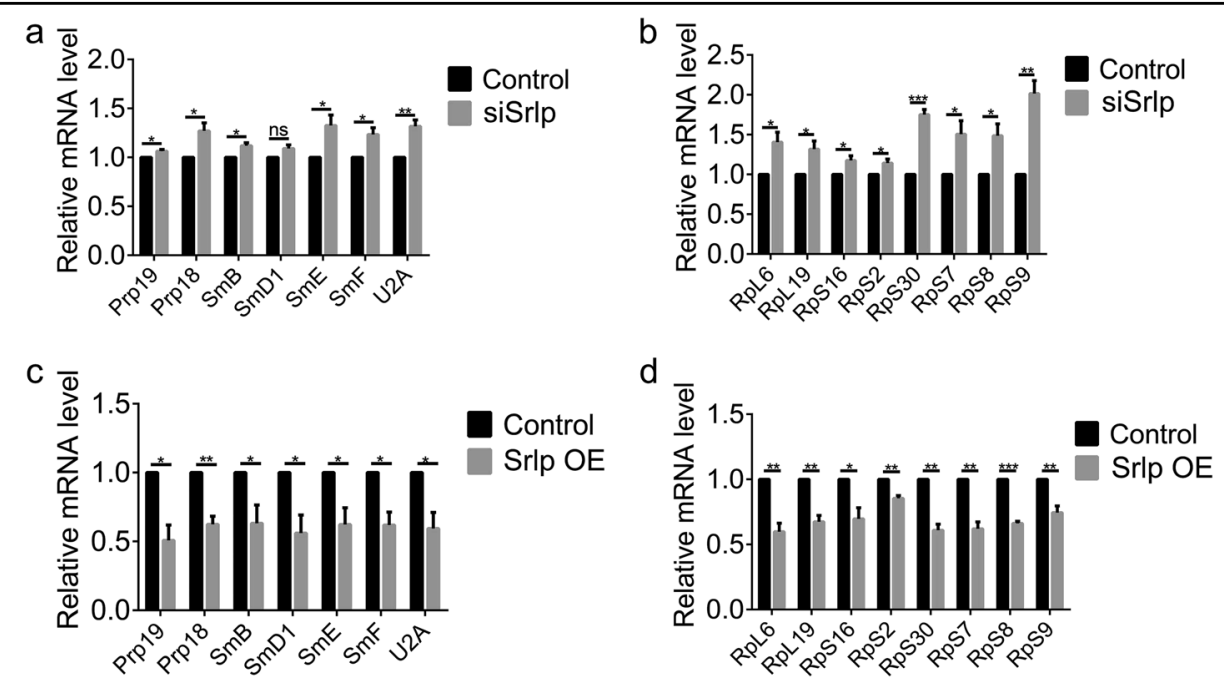

Fig. 6 Srlp regulates the expression level of major spliceosome and ribosome subunits. a Relative messenger RNA (mRNA) level of spliceosome subunits (Prp18, Prp19, SmB, SmD1, SmE, SmF, and U2A) in the control and siSrlp-684 S2 cells. b Relative mRNA levels of ribosome subunits (RpL19, RpS2, RpS7, RpS8, RpS9, RpS16, and RpS30) in control and siSrlp-684 S2 cells. c Relative mRNA level of spliceosome subunits (Prp18, Prp19, SmB, SmD1, SmE, SmF, and U2A) in control and Srlp-overexpressing S2 cells. d Relative mRNA level of ribosome subunits (RpL19, RpS2, RpS7, RpS8, RpS9, RpS16, and RpS30) in control and Srlp-overexpressing S2 cells. Student's t-test was used. ${ }^{*} P<0.05 ;{ }^{* *} P<0.01$; ${ }^{* * *} P<0.001$; n.s. not significant. Error bars represent SEM

\section{Srlp regulated the expression levels of spliceosome and ribosome subunits}

Many proteins involved in protein synthesis and degradation were identified in the screening of proteins that bound to Srlp. To further investigate whether Srlp affected the function of spliceosome and ribosome, we measured the expression level of major spliceosome and ribosome subunits. Spliceosome subunits (Prp18, Prp19, $S m B, S m D 1, S m E, S m F$, and $U 2 A)$ and ribosome subunits (RpL6, RpL19, RpS2, RpS7, RpS8, RpS9, RpS16, and $R p S 30)$ were all up-regulated in siSrlp S2 cells and downregulated in S2 cells overexpressing Srlp (Fig. 6), indicating that Srlp competitively controlled the expression level of spliceosome and ribosome, which may affect their function.

\section{Srlp regulates ribosome function via RpL6 in Drosophila}

To elucidate the potential mechanism of Srlp, we investigated whether ribosome subunits such as RpL6 were essential for self-renewal and differentiation of cells in Drosophila testes. Interestingly, RpL6 knockdown in early germ cells driven by nos-Gal4 caused tiny testes, loss of germ cells, and accumulation of cyst cells (Fig. 7a). Knockdown of RpL6 in cyst cells by tj-Gal4 led to accumulation of undifferentiated germ cells and subsequent tumor formation (Fig. 7b, c). These GSC-like germ cells exhibited the ability for self-renewal without normal maintenance of the apical hubs and CySCs (Zfh1-positive and DE-cad-positive cells existed in an abnormal pattern, and there were no Eya-positive cyst cells). Our results indicated that the $R p L 6$ gene completely mimicked the self-renewal and differentiation phenotype of the Srlp gene in GSCs in Drosophila testes.

Significantly, spliceosome subunits (Prp18, Prp19, SmB, $S m D 1, S m E, S m F$, and $U 2 A)$ and ribosome subunits (RpL19, RpS2, RpS7, RpS8, RpS9, RpS16, and RpS30) were all down-regulated in S2 cells overexpressing RpL6 (Fig. S7). Additionally, RpL6-overexpressing S2 cells exhibited proliferation ability (Fig. S8 a-d), and dramatically promoted the cell death process, as evidenced by increased ratios of apoptotic and necrotic cells (Fig. $\mathrm{S} 8 \mathrm{e}-\mathrm{g}$ ).

Next, cells overexpressing Srlp alone inhibited $R p L 6$ expression, while cells overexpressing RpL6 alone repressed Srlp expression (Fig. 8a, b). As it was obvious that RpL6 and Srlp were mutually antagonistic, we further tested whether overexpression of both RpL6 and Srlp could restore the proliferation ability and apoptosis in $\mathrm{S} 2$ cells. First, Srlp and RpL6 expression levels could be recovered in RpL6 overexpression/Srlp overexpression or Srlp overexpression/RpL6 overexpression S2 cells (Fig. 8c and Fig. S9a). Second, in cells overexpressing RpL6, the increased proliferation ability, but not the ratios of apoptotic and necrotic cells, could be partially rescued by Srlp in S2 cells (Fig. 8d-h). Furthermore, the increased proliferation in Srlp-overexpressing cells could be partially rescued by RpL6, while the ratios of apoptosis and necrosis (especially the necrosis ratio) dramatically increased (Fig. S9 b-f).

Co-transfecting S2 cells with both Srlp and RpL6 increased the expression level of Srlp and drastically suppressed the expression level of RpL6 (Fig. S10a and 

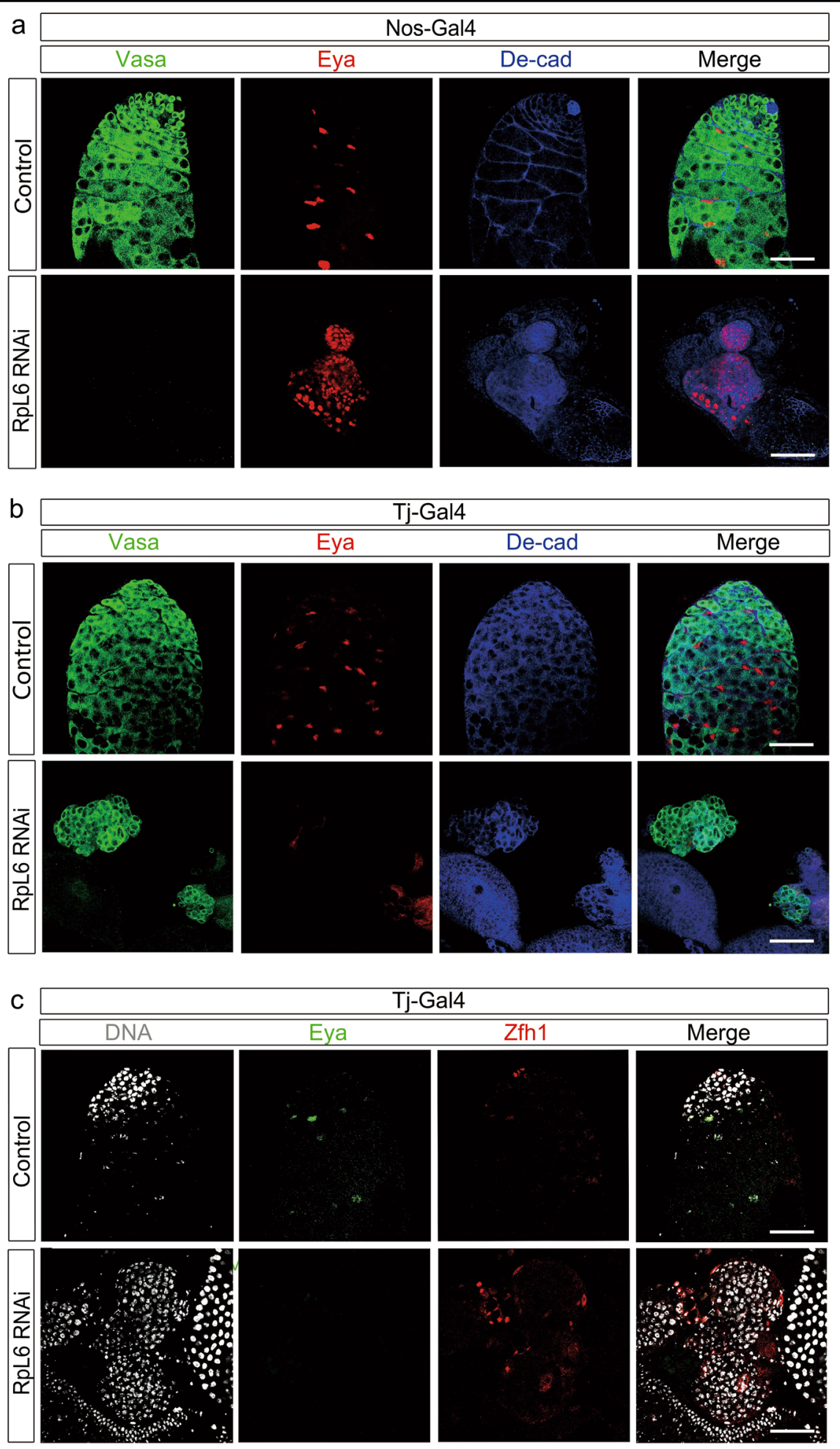

Fig. 7 Knockdown of RpL6 by nos-Gal4 and tj-Gal4 causes tiny testes and germ cell dysfunction. a Distribution of germ cells and somatic cells in the apex of the testis in the control and nos>RpL6 RNA interference (RNAi) flies. $\mathbf{b}$ Distribution of germ cells and somatic cells in the apex of the testis in the control and tj>RpL6 RNAi flies. c Distribution of germ cells and somatic cells in the apex of testis in control and tj>RpL6 RNAi flies. Immunostaining using anti-Vasa (green in $\mathbf{a}, \mathbf{b}$ ), anti-Eya (red in $\mathbf{a}, \mathbf{b}$; green in c), anti-DE-cad (blue in $\mathbf{a}, \mathbf{b}$ ), anti-Zfh1 (red in c), and Hoechst-33342 (gray in c). Scale bar: $50 \mu \mathrm{m}$ 


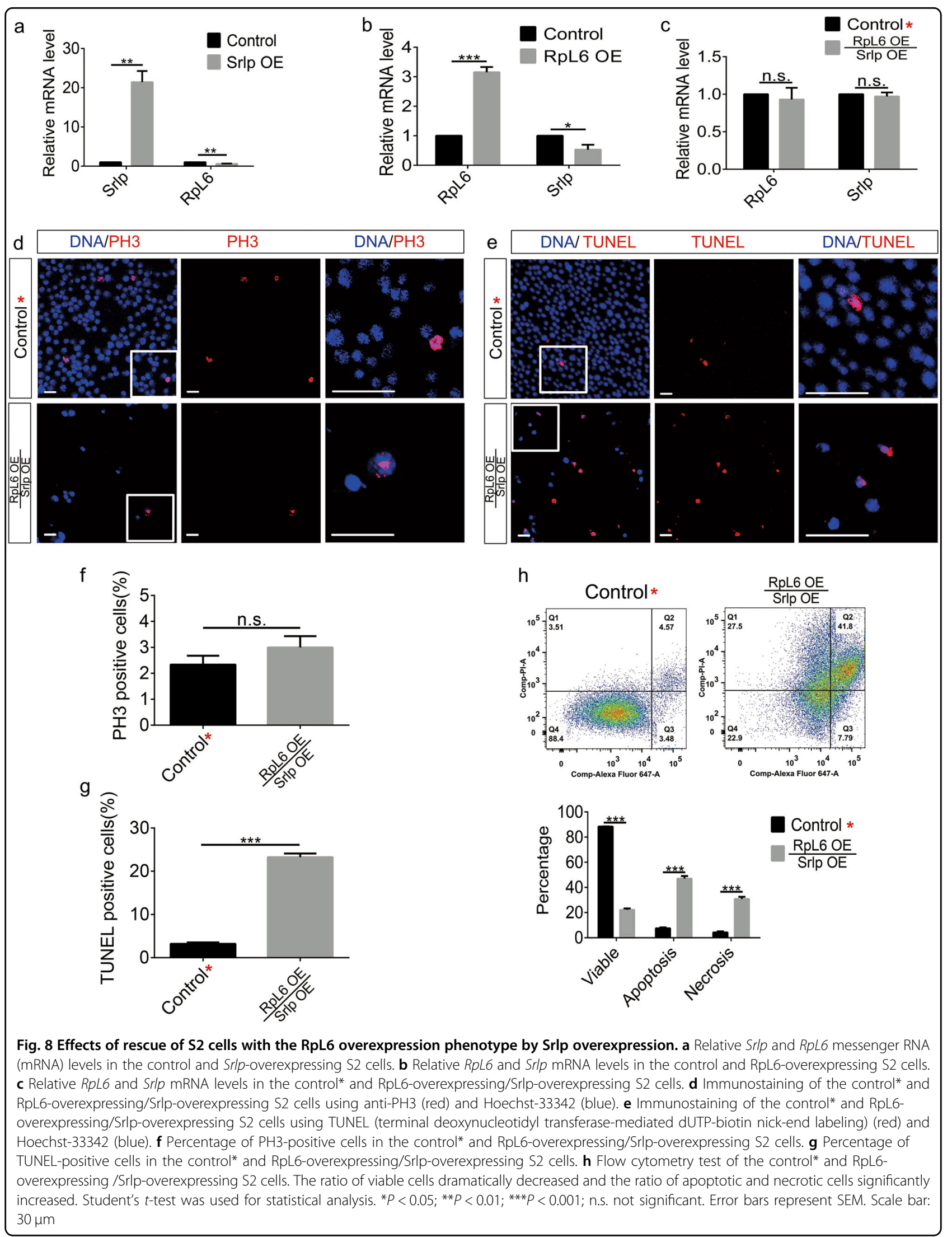


S10b). Results of the co-immunoprecipitation assay confirmed that the Srlp protein could combine with the RpL6 protein, which is consistent with LC-MS/MS data (Fig. S10c). Together, our results illustrate that RpL6 is a Srlp-binding protein and a potential target of Srlp. These results indicate that Srlp regulates the proliferation ability and cell survival via RpL6 in Drosophila testis cells.

\section{Discussion}

The stem cell niche controls normal self-renewal and differentiation of GSCs ${ }^{17,21}$. Srlp was identified as a regulatory factor of GSCs in Drosophila testes; however, its mechanism remains to be elucidated. This is the first study to investigate the function and mechanism of Srlp in Drosophila in vivo and in vitro. In this study, we used Drosophila as a model and found that Srlp is essential for the self-renewal and differentiation of GSCs in the testis and increases the proliferation and apoptosis of S2 cells. The phenotype of proliferation but not apoptosis, which was induced by silencing the Srlp gene, could be rescued by Srlp overexpression in S2 cells. Identification of Srlpbinding proteins by LC-MS/MS indicated that ribosome was enriched. Importantly, Srlp could affect the expression pattern of splicesome and ribosome subunits by competitive integration. Overexpressed RpL6 decreased the expression levels of Srlp, splicesome subunits, and ribosome subunits. This study demonstrated that Srlp regulated splicesome and ribosome function via RpL6 signals.

Previous studies have demonstrated that the regulation of protein synthesis is crucial for self-renewal and differentiation of GSCs in both fly testes and ovaries ${ }^{17,22-26}$. In the testis screen, nine ribosome proteins, four spliceosome-associated proteins, and three eIF3 complex proteins contribute to both GSC maintenance and early germ cell differentiation ${ }^{17}$. The switch from TA proliferation to differentiation in the testis is mediated by translational control of Mei-P26 and differentiation factor Bam ${ }^{15,16,27}$. Mei-P26 is a key molecule regulated by the translational machinery that controls distinct developmental programs during germ cell formation and maturation ${ }^{27,28}$. In UAS-Srlp RNAi and UAS-RpL6 RNAi testes driven by tj-Gal4, overproliferation of undifferentiated germ cells accumulate in the testes, consequently leading to tumor formation. These GSC-like cells acquired proliferation and apoptosis abilities and were devoid of distinguishable hub cells, indicating that the overproliferated GSC-like cells are selfsustained.

Spliceosome and ribosome are of great importance in the occurrence of diseases ${ }^{29,30}$. The major spliceosome is essential for mRNA processing and cell survival ${ }^{31}$. Mutation of U2A, a major spliceosome subunit, impaired the differentiation of spermatogonia, abolishing the maturation of germ cells into sperm ${ }^{18}$. Zfrp8 functions in the formation of mRNA ribonucleoprotein (RNP) complexes, and is essential for the maintenance of follicles and GSCs in the ovary ${ }^{32}$. Zfrp8 functions in the late stages of ribosome assembly and may regulate the binding of specific mRNA-RNPs to the small ribosomal subunit (RpS2), ultimately controlling their cytoplasmic localization and translation $^{33}$. Previously, a novel class of RNPs, termed regulatory RNPs, has been found to contribute to the biogenesis of small nuclear RNPs and ribosome heterogeneity $^{34}$. Specifically, the results of our study revealed that Srlp regulates splicesome and ribosome function via RpL6 signals. These findings suggest that Srlp may affect spliceosome and ribosome assembly, ultimately leading to loss of control of cell fate determination.

Previous studies have reported that apoptosis can promote the proliferation ability and the phenomenon of apoptosis-induced proliferation existed in many cell lines ${ }^{35,36}$. Moreover, apoptotic cells also had active roles for proliferation in Drosophila ${ }^{37,38}$. Srlp knockdown or Srlp-overexpressing S2 cells had a significantly higher proportion of PH3-positive and TUNEL-positive cells, indicating that Srlp is vital for the homeostasis of proliferation and apoptosis processes.

In summary, the present study mainly discussed the crosstalk between Srlp and large ribosomal subunit RpL6. Our findings demonstrated that Srlp, together with RpL6, regulated GSC self-renewal and differentiation by controlling spliceosome and ribosome function in Drosophila testis. This study may provide new insights into the pathogenic mechanism of male sterility and testicular germ cell tumor.

\section{Materials and methods \\ Fly strains and RNAi strategy}

All flies were cultured on standard corn meal food at $25^{\circ} \mathrm{C}$. The transgenic RNAi flies used in the screen were ordered from the Tsinghua Fly Center (THFC) and were from the same RNAi collection as the TRiP (Transgenic RNAi Project). Information for alleles and transgenes used in this study can be found either in FlyBase or as noted: Nos-Gal4 (BDSC, 4937), Tj-Gal4 (DGRC, 104055), UAS-Srlp RNAi (THFC, THU1733), UAS-RpL6 RNAi (THFC, THU1350).

The crosses were set and raised at room temperature $\left(25^{\circ} \mathrm{C}\right)$. Male Gal4 drivers were crossed to the transgenic UAS-RNAi virgin females. The male progenies were dissected for further function analysis.

\section{Plasmid construction}

Srlp CDS (a gift from C. Tong) was subcloned into the pUAS-attB-3xHA vector, and the CDS sequence was amplified by PCR using primers to introduce NotI (1166A, Takara, Japan) and XbaI (1093A, Takara, Japan) restriction 
sites. RpL6 CDS (a gift from C. Tong) was subcloned into the pUAS-attB vector, and the sequence was amplified by PCR using primers to introduce a V5 tag as well as NotI and $X b a \mathrm{I}$ restriction sites. Plasmid construction protocol by classical restriction ligation cloning has been described before $^{39}$. Following primers were used: pUAS-attB-3xHASrlp F: 5-ATAAGAATGCGGCCGCGATGCTTCGCAAA TTTGGAGGTC-3, R: 5-GCTCTAGACTACTTTTCGT TTGGCTTATCCTTGG-3; pUAS-attB-RpL6-V5 F: 5-A TAAGAATGCGGCCGCATGGCACCCATCGAGAAAG C-3, R: 5-GCTCTAGATTACGTAGAATCGAGACCGA GGAGAGGGTTAGGGATAGGCTTACCGAATCGCAT GCGGTGGGGGTAT-3; pUAS-attB seq primer: CCAG CAACCAAGTAAATCAA.

\section{Cell culture and transfection}

Drosophila Schneider 2 (S2) cells were obtained from Drosophila Genomics Resource Center and were grown at $28^{\circ} \mathrm{C}$ in Schneider's Drosophila medium (21720024, Gibco, USA) supplemented with $10 \%$ heat-inactivated fetal bovine serum (04-001-1ACS, Bioind, Israel). The cells were split with supplemented medium at a ratio of 1:4 every 3-4 days which has been described before ${ }^{40}$.

S2 cells were plated in the wells of six-well plates 1 day before transfection. For knockdown of Srlp, S2 cells were transfected using Lipofectamine 2000 Transfection Reagent (Lipo2000; 11668019, Invitrogen, USA). Two tubes, one containing siRNA with $250 \mu \mathrm{L}$ of opti-Minimal Essential Medium (MEM) (31985-062, Gibco, USA) and another containing a corresponding dose of Lipo2000 with $250 \mu \mathrm{L}$ of opti-MEM, were mixed and incubated for $5 \mathrm{~min}$ at room temperature. Then, the tubes were mixed and incubated for $20 \mathrm{~min}$ at room temperature. The siRNAs were designed and synthesized by GenePharma company (Suzhou, China). The siRNA information is described as follows: negative control F: 5-UUCUCCG AACGUGUCACGUTT-3, R: 5-ACGUGACACGUUCGG AGAATT-3; siSrlp-303 F: 5-GCUUACUGCAUCGCA GCUUTT-3, R: 5-AAGCUGCGAUGCAGUAAGCTT-3; siSrlp-684 F: 5-GCGUGCCCUGGACCUGAUATT-3, R: 5-UAUCAGGUCCAGGGCACGCTT-3.

For transient expression of Srlp and RpL6, S2 cells were transfected with plasmid using Effectene Transfection Reagent (301425, Qiagen, Germany). Briefly, plasmids were diluted to $100 \mu \mathrm{L}$ with buffer $\mathrm{EC}$ and $6.4 \mu \mathrm{L}$ Enhancer, vortexed, and incubated for $5 \mathrm{~min}$ at room temperature, To this, $20 \mu \mathrm{L}$ of Effectene Transfection Reagent was added for an additional $10 \mathrm{~min}$, to which $600 \mu \mathrm{L}$ of opti-MEM was mixed, and the mixture was used as the final culture medium.

The detailed plasmid and culture times are as follows:

(1) Control (negative control or ub-Gal4+pUAS-attB): culture for $48 \mathrm{~h}$.

(2) siSrlp (siRNA): culture for $48 \mathrm{~h}$.
(3) Srlp OE (ub-Gal4+pUAS-attB-3xHA-Srlp): culture for $48 \mathrm{~h}$.

(4) RpL6 OE (ub-Gal4+pUAS-attB-RpL6-V5): culture for $48 \mathrm{~h}$.

(5) Srlp OE+RpL6 OE (ub-Gal4+pUAS-attB-3xHASrlp+pUAS-attB-RpL6-V5): culture for $48 \mathrm{~h}$.

(6) Control": (a) negative control culture for $48 \mathrm{~h}$, followed by culture with ub-Gal4+pUAS-attB for $48 \mathrm{~h}$; (b) ub-Gal4+pUAS-attB culture for $48 \mathrm{~h}$, followed by negative control culture for $48 \mathrm{~h}$; and (c) ub-Gal4+pUAS-attB culture for $96 \mathrm{~h}$ (corresponding to the experimental group).

(7) siSrlp/Srlp OE: siSrlp culture for $48 \mathrm{~h}$, followed by culture of Srlp OE for $48 \mathrm{~h}$.

(8) Srlp OE/ siSrlp: culture of Srlp OE cells for $48 \mathrm{~h}$, followed by culture of siSrlp cells for $48 \mathrm{~h}$.

(9) Srlp OE/RpL6 OE: Srlp OE culture for $48 \mathrm{~h}$, followed by culture of RpL6-overexpressing cells for $48 \mathrm{~h}$.

(10) RpL6 OE/Srlp OE: RpL6 OE culture for $48 \mathrm{~h}$, followed by Srlp OE culture for $48 \mathrm{~h}$.

\section{Quantitative reverse transcription-PCR}

Total RNA was extracted using Trizol reagent (9108, Takara, Japan). Complementary DNA was synthesized using Prime Script RT Reagent Kit (RR037A, Taraka, Japan), and qRT-PCR was performed using SYBR Premix Ex Taq (RR420A, Takara, Japan). Glyceraldehyde 3phosphate dehydrogenase (GAPDH) was amplified as an internal standard. Fold changes were calculated using the standard curve according to the manufacturer's protocol. Each experiment was independently repeated three times. All primers used for qRT-PCR are listed in Table S2.

\section{Immunofluorescence and antibodies}

Fly testes were dissected in $1 \times$ phosphate-buffered saline (PBS) and fixed for $30 \mathrm{~min}$ in $4 \%$ paraformaldehyde. After washing three times in $1 \times$ PBS with $0.1 \%$ Triton $\mathrm{X}-100$ (PBST) and blocking for $1 \mathrm{~h}$ in $5 \%$ bovine serum albumin, the samples were incubated with primary antibodies overnight at $4{ }^{\circ} \mathrm{C}$. After washing three times for $10 \mathrm{~min}$ in $0.1 \%$ PBST, the samples were incubated for $1 \mathrm{~h}$ with secondary antibodies at room temperature followed by three times washing in $0.1 \%$ PBST. Testes were then stained with Hoechst-33342 (1.0 mg/mL, Invitrogen) for 5 min before mounting. Images were captured on an LSM710 Zeiss confocal microscope and processed using Adobe Photoshop CS5 software. Drosophila S2 cells were cultured for $24 \mathrm{~h}$, and immunostaining was carried out in the culture dish according to the protocols described above.

The antibodies used were as follows: mouse anti-Eya (Developmental Studies Hybridoma Bank, 1:20); rat antiDE-cadherin (Developmental Studies Hybridoma Bank, 1:20); mouse anti-FasIII (Developmental Studies 
Hybridoma Bank, 1:50); rabbit anti-Vasa (a gift from C. Tong, 1:1000); rat anti-Zfh1 (a gift from C. Tong, 1:2000); rabbit anti-PH3 (53348, Cell Signaling Technology, 1:1000); anti-V5 (R960-25, Invitrogen, 1:500); and rabbit anti-HA (3724, Cell Signaling Technology, 1:1000). Secondary antibodies conjugated to A488, Cy3, A594, or A647 (Molecular Probes and Jackson Immunologicals) were diluted at 1:1000.

\section{TUNEL assay}

Cell apoptosis was determined using the TUNEL assay according to the manufacturer's protocols. The TUNEL BrightRed Apoptosis Detection Kit was obtained from Vazyme (A113, Nanjing, China).

\section{Flow cytometry assay}

Flow cytometry was performed using an Annexin VAlexa Fluor 647/propidium iodide (PI) Apoptosis Assay Kit (FMSAV647-100, FcMACS, Nanjing, China). After transfection for $48 \mathrm{~h}, \mathrm{~S} 2$ cells were washed with ice-cold PBS. Different cell groups were stained with the apoptosis detection kit according to the manufacturer's instructions. Cells $\left(1 \times 10^{6}\right.$ cells per well $)$ from each sample were suspended and incubated in $250 \mu \mathrm{L}$ of binding buffer. Then, $100 \mu \mathrm{L}$ of the cell suspension was incubated with $5 \mu \mathrm{L}$ of Annexin V-Alexa Fluor 647 and $10 \mu \mathrm{L}$ of PI for $15 \mathrm{~min}$ at room temperature in the dark. Then, $200 \mu \mathrm{L} 1 \times$ PBS was added into each sample and mixed. The samples were then analyzed using FACScan flow cytometry (BD Biosciences, San Jose, CA, USA). Data were calculated at different intervals. Flow cytometry assay was performed with at least three independent experiments.

\section{Cell viability assay}

CCK-8 assay (CK04-3000T, DOJINDO, Japan) was utilized to assess Drosophila S2 cell viability according to the manufacturer's protocols. Briefly, transfected S2 cell were transferred to 96-well plates (3000 cells per well), and incubated in 10\% CCK-8 reagent that was diluted in Schneider's Drosophila medium at $37^{\circ} \mathrm{C}$ for $1 \mathrm{~h}$. After transfection at 0,24 , and $48 \mathrm{~h}$, the absorbance in each well was evaluated at $450 \mathrm{~nm}$ (Multiskan GO, Thermo Scientific, Waltham, USA). All experiments were repeated at least three times.

\section{LC-MS/MS}

Protein extraction and digestion were performed according to the methods described before ${ }^{41}$. The protein digests were separated using a 10 min elution gradient at a flow rate of $2 \mu \mathrm{L}$ per min in an Eksigent nanoLC-Ultra 2D system (AB SCIEX). A C18 reversed phase chromatographic column $(75 \mu \mathrm{m} \times 15 \mathrm{~cm}, 3 \mu \mathrm{m}, 120 \AA$, ChromXP Eksigent) was used as the analytical column. MS/MS scan was performed by tripleTOF5600 system (AB SCIEX).

\section{Bioinformatics analysis}

HA-Srlp protein in control and Srlp-overexpressing S2 cells are pulled down by HA beads, and followed by LCMS/MS identification. Drosophila melanogaster proteins identified in Srlp-overexpression group and not in control group were considered as candidates of Srlp-binding proteins. To analyze the functional characteristics of Srlpbinding proteins, the OmicsBean data integration analysis platform was used to perform GO functional annotation, KEGG Pathway, and PPI analysis.

\section{Statistical analysis}

Experiments were repeated at least three times. The quantitative results are presented as mean \pm standard error of mean (SEM). The data were evaluated for statistical differences using Student's $t$-test and one-way analysis of variance by Graphpad Software (https://www. graphpad.com/) with " $P<0.05$; ${ }^{* *} P<0.01$; ${ }^{* * * *} P<0.001$.

\section{Acknowledgements \\ The authors wish to thank all study participants, research staff, and students who assisted with this work. We would like to thank Chao Tong from Life Sciences Institute, Zhejiang University, for sharing reagents and stocks. We are grateful to the THFC, BDSC, DGRC, and DHSB for providing fly strains and antibodies. This work was supported by National Natural Science Foundation of China (31701298, 81803505), Natural Science Foundation of Jiangsu Province (BK20170562, BK20170564), Key Research Foundation of Zhenjiang Social Development (SH2016028, SH2017013, SH2017020, SH2018065), Suzhou Key Laboratory of Male Reproduction Research (SZS201718), Basic Program of Nanjing Medical University (2017NJMU163), Suzhou Key Medical Center (SZZX201505), Suzhou Introduced Project of Clinical Medical Expert Team (SZYJTD201708), and Jiangsu Provincial Medical Innovation Team (CXTDB2017013).}

\section{Author details}

'Department of Gynecology, The Affiliated Hospital of Jiangsu University, Jiangsu University, Zhenjiang Jiangsu 212001, China. ${ }^{2}$ Reproductive Sciences Institute of Jiangsu University, Zhenjiang Jiangsu 212001, China. ${ }^{3}$ Department of Clinical Pharmacy, the Affiliated Hospital of Jiangsu University, Jiangsu University, Zhenjiang Jiangsu 212001, China. ${ }^{4}$ Center for Reproduction and Genetics, Suzhou Municipal Hospital, The Affiliated Suzhou Hospital of Nanjing Medical University, Suzhou Jiangsu 215002, China. ${ }^{5}$ Center for Reproduction, The Fourth People's Hospital of Zhenjiang, Zhenjiang Jiangsu 212013, China. ${ }^{6}$ Department of Clinical Medicine, China Medical University, Shenyang Liaoning 110001, China. ' State Key Laboratory of Reproductive Medicine, Department of Histology and Embryology, Nanjing Medical University, Nanjing Jiangsu 211166, China. ${ }^{8}$ Department of Immunology and Jiangsu Key Laboratory of Medical Science and Laboratory Medicine, School of Medicine, Jiangsu University, Zhenjiang Jiangsu 212013, China

\section{Authors' contributions}

J.Y. and X.C. designed the study, coordinated the experiments, and wrote the manuscript. Y.Y., C.Q., and X.L. contributed to the design of the experiments on plasmid construction, cell culture, transfection, immunofluorescence, and provided critical inputs for manuscript. J.Y., J.F., B.Z., Y.L., and Q.Z. performed the majority of the experiments, provided critical inputs, and contributed to the preparation and editing of the manuscript. D.Z. and B.X. contributed to western blot. Y.Y. and W.C. performed flow cytometry and cell viability assay. Y.D. and M.W. performed the experiments of qRT-PCR. C.S. and Z.H. performed some of the experiments and provided conceptual inputs for the paper. X.L. performed the statistical analysis. X.H., X.Y.H., H.L., and Q.S. provided advices and reviewed the manuscript.

Conflict of interest

The authors declare that they have no conflict of interest. 


\section{Publisher's note}

Springer Nature remains neutral with regard to jurisdictional claims in published maps and institutional affiliations.

Supplementary Information accompanies this paper at (https://doi.org/ 10.1038/s41419-019-1527-z).

Received: 10 October 2018 Revised: 11 March 2019 Accepted: 14 March 2019

Published online: 01 April 2019

\section{References}

1. Davies, E. L. \& Fuller, M. T. Regulation of self-renewal and differentiation in adult stem cell lineages: lessons from the Drosophila male germ line. Cold Spring Harb. Symp. Quant. Biol. 73, 137-145 (2008).

2. de Cuevas, M. \& Matunis, E. L. The stem cell niche: lessons from the Drosophila testis. Development 138, 2861-2869 (2011).

3. Losick, V. P., Morris, L. X., Fox, D. T. \& Spradling, A. Drosophila stem cell niches: a decade of discovery suggests a unified view of stem cell regulation. Dev. Cell 21, 159-171 (2011).

4. Carbonell, A., Pérez-Montero, S., Climent-Cantó, P., Reina, O. \& Azorín, F. The germline linker histone $\mathrm{dBigH1}$ and the translational regulator Bam form a repressor loop essential for male germ stem cell differentiation. Cell Rep. 21, 3178-3189 (2017)

5. Leatherman, J. L. \& Dinardo, S. Germline self-renewal requires cyst stem cells and stat regulates niche adhesion in Drosophila testes. Nat. Cell Biol. 12, 806-811 (2010).

6. Lim, J. G. \& Fuller, M. T. Somatic cell lineage is required for differentiation and not maintenance of germline stem cells in Drosophila testes. Proc. Natl. Acad. Sci. USA 109, 18477-18481 (2012).

7. Kiger, A. A., Jones, D. L., Schulz, C., Rogers, M. B. \& Fuller, M. T. Stem cell selfrenewal specified by JAK-STAT activation in response to a support cell cue. Science 294, 2542-2545 (2001)

8. Tulina, N. \& Matunis, E. Control of stem cell self-renewal in Drosophila spermatogenesis by JAK-STAT signaling. Science 294, 2546-2549 (2001).

9. Zhang, Z., LV, X., Jiang, J., Zhang, L. \& Zhao, Y. Dual roles of Hh signaling in the regulation of somatic stem cell self-renewal and germline stem cell maintenance in Drosophila testis. Cell Res. 23, 573-576 (2013).

10. Kawase, E., Wong, M. D., Ding, B. C. \& Xie, T. Gbb/Bmp signaling is essential for maintaining germline stem cells and for repressing bam transcription in the Drosophila testis. Development 131, 1365-1375 (2004).

11. Bunt, S. M. \& Hime, G. R. Ectopic activation of Dpp signalling in the male Drosophila germline inhibits germ cell differentiation. Genesis 39, 84-93 (2004).

12. Shivdasani, A. A. \& Ingham, P. W. Regulation of stem cell maintenance and transit amplifying cell proliferation by tgf-beta signaling in Drosophila spermatogenesis. Curr. Biol. 13, 2065-2072 (2003).

13. Eun, S. H. et al. MicroRNAs downregulate Bag of marbles to ensure proper terminal differentiation in the Drosophila male germline. Development $\mathbf{1 4 0}$ 23-30 (2013).

14. Gönczy, P., Matunis, E. \& DiNardo, S. bag-of-marbles and benign gonial cell neoplasm act in the germline to restrict proliferation during Drosophila spermatogenesis. Development 124, 4361-4371 (1997).

15. Chen, D. et al. Three RNA binding proteins form a complex to promote differentiation of germline stem cell lineage in Drosophila. PLoS Genet. 10, e1004797 (2014).

16. Insco, M. L. et al. A self-limiting switch based on translational control regulates the transition from proliferation to differentiation in an adult stem cell lineage. Cell Stem Cell 11, 689-700 (2012).

17. $\mathrm{Yu}, \mathrm{J}$. et al. Protein synthesis and degradation are essential to regulate germline stem cell homeostasis in Drosophila testes. Development $\mathbf{1 4 3}$ 2930-2945 (2016).

18. Wu, H. et al. Major spliceosome defects cause male infertility and are associated with nonobstructive azoospermia in humans. Proc. Natl. Acad. Sci. USA 113, 4134-4139 (2016).
19. $\mathrm{Yu}$, J. et al. Identification of seven genes essential for male fertility through a genome-wide association study of non-obstructive azoospermia and RNA interference-mediated large-scale functional screening in Drosophila. Hum. Mol. Genet. 24, 1493-1503 (2015).

20. Yan, D. et al. A regulatory network of Drosophila germline stem cell selfrenewal. Dev. Cell 28, 459-473 (2014).

21. Xie, T. Control of germline stem cell self-renewal and differentiation in the Drosophila ovary: concerted actions of niche signals and intrinsic factors. Wiley Interdiscip. Rev. Dev. Biol. 2, 261-273 (2013).

22. Sanchez, C. G. et al. Regulation of ribosome biogenesis and protein synthesis controls germline stem cell differentiation. Cell Stem Cell 18, 276-290 (2016).

23. Lee, J. Y., Chen, J. Y., Shaw, J. L. \& Chang, K. T. Maintenance of stem cell niche integrity by a novel activator of integrin signaling. PLoS Genet. 12, e1006043 (2016).

24. Shen, R., Weng, C., Yu, J. \& Xie, T. elF4A controls germline stem cell self-renewal by directly inhibiting BAM function in the Drosophila ovary. Proc. Natl. Acad. Sci. USA 106, 11623-11628 (2009).

25. Spradling, A., Fuller, M. T., Braun, R. E. \& Yoshida, S. Germline stem cells. Cold Spring Harb. Perspect. Biol. 3, a002642 (2011).

26. Wang, Z. \& Lin, H. Nanos maintains germline stem cell self-renewal by preventing differentiation. Science 303, 2016-2019 (2004).

27. Li, Y., Maines, J. Z., Tastan, O. Y., McKearin, D. M. \& Buszczak, M. Mei-P26 regulates the maintenance of ovarian germline stem cells by promoting BMP signaling. Development 139, 1547-1556 (2012).

28. Neumüller, R. A. et al. Mei-P26 regulates microRNAs and cell growth in the Drosophila ovarian stem cell lineage. Nature 454, 241-245 (2008).

29. Singh, R. K. \& Cooper, T. A. Pre-mRNA splicing in disease and therapeutics. Trends Mol. Med. 18, 472-482 (2012).

30. Pelava, A., Schneider, C. \& Watkins, N. J. The importance of ribosome production, and the 5S RNP-MDM2 pathway, in health and disease. Biochem. Soc. Trans. 44, 1086-1090 (2016).

31. Gruss, O. J., Meduri, R., Schilling, M. \& Fischer, U. UsnRNP biogenesis: mechanisms and regulation. Chromosoma 126, 577-593 (2017).

32. Minakhina, S., Changela, N. \& Steward, R. Zfrp8/PDCD2 is required in ovarian stem cells and interacts with the piRNA pathway machinery. Development 141, 259-268 (2014).

33. Minakhina, S., Naryshkina, T., Changela, N., Tan, W. \& Steward, R. Zfrp8/PDCD2 interacts with RpS2 connecting ribosome maturation and gene-specific translation. PLoS One 11, e0147631 (2016).

34. Poole, A. R., Vicino, I., Adachi, H., Yu, Y. T. \& Hebert, M. D. Regulatory RNPs: a novel class of ribonucleoproteins that potentially contribute to ribosome heterogeneity. Biol. Open 6, 1342-1354 (2017).

35. Huang, Q. et al. Caspase 3-mediated stimulation of tumor cell repopulation during cancer radiotherapy. Nat. Med. 17, 860-866 (2011).

36. Ryoo, H. D. \& Bergmann, A. The role of apoptosis-induced proliferation for regeneration and cancer. Cold Spring Harb. Perspect. Biol. 4, a008797 (2012).

37. Clavier, A., Rincheval-Arnold, A., Baillet, A., Mignotte, B. \& Guénal, I. Two different specific JNK activators are required to trigger apoptosis or compensatory proliferation in response to Rbf1 in Drosophila. Cell Cycle 15, 283-294 (2016).

38. Dichtel-Danjoy, M. L. et al. Drosophila p53 isoforms differentially regulate apoptosis and apoptosis-induced proliferation. Cell Death Differ. 20, 108-116 (2013).

39. Zitzmann, J. et al. Single-cell cloning enables the selection of more productive Drosophila melanogaster S2 cells for recombinant protein expression. Biotechnol. Rep. (Amst.) 19, e00272 (2018).

40. Uribe, E., Venkatesan, M., Rose, D. R. \& Ewart, K. V. Expression of recombinant Atlantic salmon serum C-type lectin in Drosophila melanogaster Schneider 2 cells. Cytotechnology 65, 513-521 (2013).

41. Katayama, H., Nagasu, T. \& Oda, Y. Improvement of in-gel digestion protocol for peptide mass fingerprinting by matrix-assisted laser desorption/ionization time-of-flight mass spectrometry. Rapid Commun. Mass Spectrom. 15, 1416-1421 (2001). 